\title{
A case of $\left[{ }^{68} \mathrm{Ga}\right] \mathrm{Ga}-\mathrm{FAPI}-46$-avid and $\left[{ }^{18} \mathrm{~F}\right] \mathrm{F}-\mathrm{FDG}$-negative COVID-19 pneumonia sequelae
}

\author{
Silvi Telo ${ }^{1}$ (1) $\cdot$ Andrea Farolfi ${ }^{1} \cdot$ Paolo Castellucci $^{1} \cdot$ Filippo Antonacci $^{2} \cdot$ Piergiorgio Solli $^{2} \cdot$ Cristina Mosconi $^{3}$. \\ Stefano Fanti ${ }^{1} \cdot$ Roberto Agosti ${ }^{4}$. Joshua James Morigi ${ }^{5} \cdot$ Cristina Nanni $^{1}$
}

Received: 16 December 2021 / Accepted: 6 February 2022 / Published online: 18 February 2022

(c) The Author(s), under exclusive licence to Springer-Verlag GmbH Germany, part of Springer Nature 2022

A 72-year-old woman with COVID-19 respiratory syndrome symptoms underwent a chest CT scan (CT) for investigation. The CT detected a lung mass with no evidence of pneumonia (Fig. a); however, an RT-PCR nasopharyngeal swab (PCR) resulted positive for COVID-19 infection. Subsequent high-resolution CT (HRCT) performed 5 days later identified diffuse, bilateral peripheral ground-glass-opacities (GGO) (Fig. b, red arrow). After returning a negative PCR,

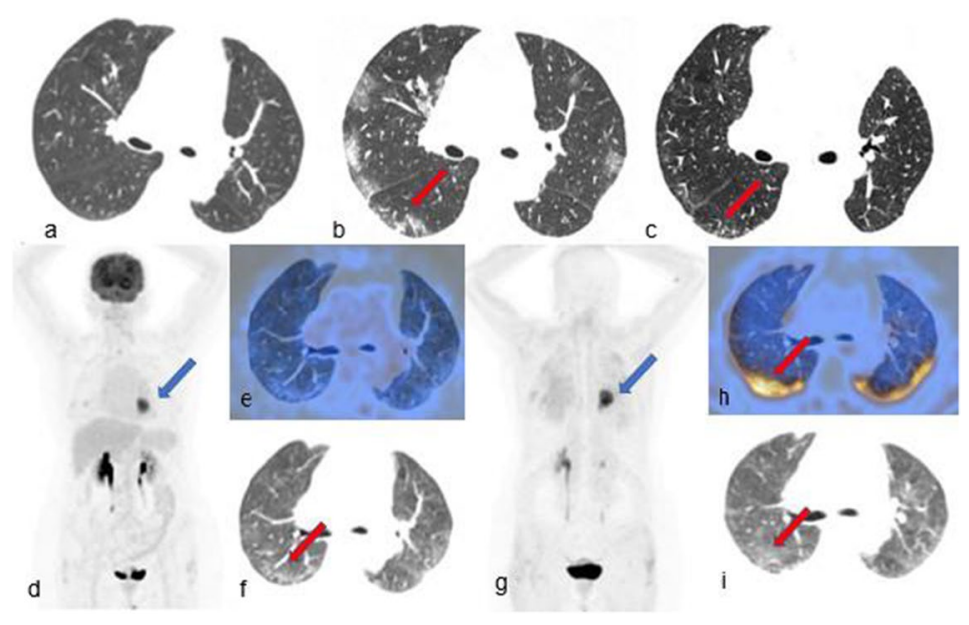

This article is part of the Topical Collection on Infection and inflammation

Silvi Telo

silvi.telo@gmail.com

1 Nuclear Medicine Department, IRCCS Azienda Ospedaliero-Universitaria Di Bologna, Bologna, Italy

2 Thoracic Surgery Department, IRCCS Azienda Ospedaliero-Universitaria Di Bologna, Bologna, Italy

3 Radiology Department, IRCCS Azienda Ospedaliero-Universitaria Di Bologna, Bologna, Italy

4 Pathology Department, IRCCS Azienda Ospedaliero-Universitaria Di Bologna, Bologna, Italy

5 PET/CT Unit, Department of Medical Imaging, Royal Darwin Hospital, Darwin, Australia $\left[{ }^{18} \mathrm{~F}\right] \mathrm{F}-\mathrm{FDG}$ PET/CT (Fig. d, e, f) and $\left[{ }^{68} \mathrm{Ga}\right] \mathrm{Ga}-\mathrm{FAPI}-46$ PET/CT (Fig. g, h, i) were performed within 6 days. Both scans demonstrated intense tracer-uptake of the lung mass (blue arrows) which was proven to be an adenocarcinoma at following surgical excision. $\left[{ }^{68} \mathrm{Ga}\right] \mathrm{Ga}-\mathrm{FAPI}-46 \mathrm{PET} / \mathrm{CT}$ also demonstrated diffuse bilateral posterior lung uptake (Fig. $h$, red arrow) that was not evident on the $\left[{ }^{18} \mathrm{~F}\right] \mathrm{F}-\mathrm{FDG}$ PET/ CT. This finding corresponded to the GGO detected both at HRCT and PET/CT and to a pattern of crazy-paving with septal thickening at a follow-up HRCT(Fig. c, red arrow).

FAPI PET/CT targets the fibroblast activation protein (FAP) which is overexpressed in activated fibroblasts highly present in tumor stroma, but also in benign processes such as fibrosis [1]. 
Interestingly, GGO in this patient demonstrated no significant $\left[{ }^{18} \mathrm{~F}\right] \mathrm{F}-\mathrm{FDG}$-uptake $(\mathrm{SUV} \max =3.3$; liver SUV$\max =4.5$; blood-pool SUVmax $=3.7$ ) paired with increased $\left[{ }^{68} \mathrm{Ga}\right] \mathrm{Ga}$-FAPI-46 uptake (SUVmax $=8.2$ ), suggesting no active inflammation with persisting activated fibroblasts.

Pulmonary fibrosis was reported both in COVID-19 patients and pathological specimens $[2,3]$.

In this case, fibrosis was not identified at HRCT nor reviewing surgical slides of previously resected healthy lung parenchyma margins surrounding lung cancer. HRCT demonstrated instead crazy-paving with superimposed interlobular-septal thickening which can be a feature of lung fibrosis [4], with persistence 3 months after.

To the best of our knowledge, this is the first reported case of FAPI-avid COVID-19 lung infection sequelae. Although lung fibrosis/COVID-19 correlation needs to be investigated in further studies, developing tools to estimate the burden of pulmonary fibrosis or to discriminate between inflammation and fibrosis in patients with lung infections such as COVID19 could be helpful to predict disease long term effects [5].

Author contribution Silvi Telo, Cristina Nanni, and Andrea Farolfi contributed to the study conception and design. Material preparation, data collection, and analysis were performed by Silvi Telo, Cristina Nanni, and Andrea Farolfi. The first draft of the manuscript was written by Silvi Telo and all authors commented on previous versions of the manuscript. All authors read and approved the final manuscript.

\section{Declarations}

Ethics approval This study was performed in line with the principles of the Declaration of Helsinki. Approval was granted by the Ethics Committee (EudraCT 2020-005549-17).
Consent to participate The patient gave written consent for the use of anonymous data for research purpose (CE approval: EudraCT 2020005549-17).

Conflict of interest The authors declare no competing interests.

\section{References}

1. Lindner T, Loktev A, Giesel F, Kratochwil C, Altmann A, Haberkorn U. Targeting of activated fibroblasts for imaging and therapy. EJNMMI Radiopharm Chem. 2019;4(1):16. https://doi.org/10. 1186/s41181-019-0069-0.

2. George PM, Wells AU, Jenkins RG. Pulmonary fibrosis and COVID-19: the potential role for antifibrotic therapy. Lancet Respir Med. 2020;8(8):807-15. https://doi.org/10.1016/S22132600(20)30225-3.

3. Zhao L, Wang X, Xiong Y, Fan Y, Zhou Y, Zhu W. Correlation of autopsy pathological findings and imaging features from 9 fatal cases of COVID-19 pneumonia. Medicine (Baltimore). 2021;100(12): e25232. https://doi.org/10.1097/MD.0000000000 025232 .

4. De Wever W, Meersschaert J, Coolen J, Verbeken E, Verschakelen JA. The crazy-paving pattern: a radiological-pathological correlation. Insights Imaging. 2011;2(2):117-32. https://doi.org/10.1007/ s13244-010-0060-5.

5. Schmidkonz C, Rauber S, Atzinger A, Agarwal R, Götz TI, Soare A, Cordes M, Prante O, Bergmann C, Kleyer A, Ritt P, Maschauer S, Hennig P, Toms J, Köhner M, Manger B, Stone JH, Haberkorn U, Baeuerle T, Distler JHW, Agaimy A, Kuwert T, Schett G, Ramming A. Disentangling inflammatory from fibrotic disease activity by fibroblast activation protein imaging. Ann Rheum Dis. 2020;79(11):1485-91. https://doi.org/10.1136/ annrheumdis-2020-217408.

Publisher's note Springer Nature remains neutral with regard to jurisdictional claims in published maps and institutional affiliations. 